(d) That, from immunity tests alone, there is sufficient evidence to regard Bacillus parabotulinus as being distinct from Bacillus botulinus.

Other tests, though not so comprehensive as that given above, have given the same results.

\title{
CONCLUSIONS.
}

I. That Bacillus parabotulinus produces a true toxin, i.e., a soluble exotoxin obtainable by filtration, causing symptoms only after a definite period of incubation, and capable of inducing the formation of an antitoxin.

2. That, though the toxins of Bacillus parabotulinus and of Bacillus botulinus are identical in their action, the antitoxin to the one does not protect against the other, and vice versa.

3. As it has been shown previously that Bacillus parabotulinus differs from Bacillus botulinus (Types A and B) both morphologically and culturally, and it is now demonstrated by toxinantitoxin tests to be distinct, the specific identity of Bacillus parabotulinus is claimed.

\section{CRITICISM OF DR. SCHARRER'S PAPER ON BOVINE STAGGERS.}

By W. H. Andrews, D.Sc., M.R.C.V.S., Senior Research Officer Onderstepoort.

THE isolation of the toxic principle of Matricaria nigellæfolia, and the study of its actions in the bovine body, would have great theoretical interest, in view of the nature of the intoxication and the fact that all domestic animals other than bovines appear to be relatively very insensitive. The discovery of a successful method of treatment would have considerable practical importance for many farmers in the Natal Midlands. The claims advanced in this connection by Dr. Scharrer in the article which appeared in the June number of this Journal, however, appear to invite criticism, and some of his statements can hardly be allowed to pass unchallenged.

In the first place, it is regrettable that the paper contains no description of any of the methods employed by the author (with the exception of a few remarks on chemical tests and procedures, to which further reference is made later) and no details whatever are given with respect to any experimental case of the intoxication. In no case is one able to consider the methods employed, the precautions against possible error which were adopted, and the course and nature of the resulting intoxication. This complete absence of definite experimental data deprives the reader of any opportunity of forming his own estimate of the grounds on which conclusions are based, and therefore of the justification for, or 
value of, those conclusions. The absence of such data is particularly to be deplored on account of what one must call the startling nature of some of the statements to be found in the article.

The paper contains a number of statements which do not agree at all with the observations made by me during the course of the investigations described in an article which appeared in this Journal (in December I921), but it is of course impossible to ascertain whether the number and nature of the author's observations justify the statements.

In his introduction he refers to the appearance of symptoms of gastro-enteritis, nephritis, and irritation of sexual organs soon after the consumption of the plant by any of the ordinary domestic animals. In feeding tests carried out in I9I9-20 on six horses, three sheep, two goats, and two pigs, as well as on rabbits and guinea-pigs, it was never possible to detect any signs at all of these alleged effects. About half of the experimental cattle showed certain symptoms of abdominal pain associated with constipation (diarrhcea in one case only), but the condition was of short duration and not one of any severity, and certainly no signs of irritation of the sexual organs were ever observed. The remaining cattle, and all the experimental animals of other species, showed no immediate effects at all.

Again, the author refers to "the loss of condition with relatively good appetite which is observed in the disease." A very large number of natural cases came under observation during the period I9I8-20, and it can be stated quite emphatically that a noticeable loss of condition prior to the appearance of symptoms is not the rule in naturally acquired matricaria intoxication. Once symptoms have appeared the loss of flesh is usually very rapid, but when the signs of illness first appear the animals are commonly in the same condition as the rest of the herd; they are very often in fairly good condition, and they may be quite fat.

This feature of the natural intoxication was so clearly and definitely shown that, in connection with the investigations of I9I8-20, it was considered necessary to comment on the loss of condition apparent in the experimental animals during the course of the feeding tests (see this Journal, December I921, pp. 295 and 301 ), and it was attributed to certain artificial conditions of the experiments-partial starvation and the consumption of matricaria in larger amounts than are taken naturally. Moreover, cases were recorded (p. 295) in which considerable improvement in condition was noted during the period elapsing between the cessation of experimental feeding and the appearance of symptoms.

The author states further that " pushing animals in good condition have eaten in a short period a very large dose of the plant, and the attack is therefore very acute." With reference to this one may state from experience that, under natural conditions, cases of various types, and following courses of very different duration, may be seen in animals which are, at the commencement of the attack, in good condition.

It has already been stated that loss of condition prior to the onset of symptoms was, in our experience, only clearly shown in experimental cases, in which the daily consumption of matricaria 
(during the feeding test) was most probably unnaturally great. It may be added that the course of these experimental cases was certainly more acute than that commonly seen in natural outbreaks and in many animals acquiring the intoxication naturally and developing symptoms when in good condition.

The author states that a larger dose is tolerated if its administration be spread over a long period. Reference to the article already mentioned (pp. 300-30r) will show that our observations suggested that prolongation of the period of administration (without unduly prolonging the interval between successive doses) probably causes a smaller total amount to become effective. More recent experiments have tended to confirm this, although they are at present incomplete, and the view stated has not yet been definitely proved to be correct, but it would be most interesting to examine $\mathrm{Dr}$ Scharrer's evidence in support of his assertion.

Under the heading Post-mortem, the author states that the vessels of the pia mater were always found to be distinctly engorged. The writer must confess that he was never able to detect any such congestion, and that he accepts most of such statements (which have been made in connection with almost every condition characterised by nervous symptoms) with considerable reserve.

In connection with the macroscopic lesions of the disease, it is regrettable that Dr Scharrer has omitted to define the nature of " the distinct alteration in colour and consistence" which is said to affect the brain.

The author refers to the connection between glucoside formation and the maturing of plants, and asserts that bovine staggers occurs mostly at the end of autumn, a few weeks after the plant has matured. This statement is certainly incorrect. Observation of the natural outbreaks occurring in $1918-20$, and the records relating to past outbreaks which were collected from all available sources in Natal, as a result of long and careful enquiry, have shown quite clearly that the spring is the season during which the majority of outbreaks have occurred. Incidentally, one may remark that much of the plant has matured long before the end of the autumn.

One does not know if Dr Scharrer has any definite evidence that the fully-matured plant is more toxic, and in the absence of any conclusive evidence one would not venture to make any dogmatic assertion on the point, but there are ample grounds for stating that the young and immature plant has proved in feeding experiments to be very decidedly toxic. Some of our tethered animals (see this Journal, December I92I) developed acute symptoms after consuming relatively small amounts of very young and immature matricaria.

With reference to the statement that the period of incubation may be as long as twelve to fifteen weeks, one would not venture to deny that it may be as long as that, or even longer, under particular conditions, but one may emphasise the importance of the conditions under which the animals were kept during the supposed incubation period. There is reason to believe that at least some of the author's experimental cattle were kept, during that important period, in paddocks in which matricaria was actually growing.

The statement, repeated on several occasions, that the intoxication 
could be prevented or delayed by giving food rich in albumen, leads one naturally to enquire whether the author ever actually demonstrated this as a fact. Did he, with proper controls, actually cause bovines to consume quantities of matricaria known to be lethal, and prevent or delay the usual effects by the administration of protein in any form? Only the successful performance of such an experiment could furnish justification for the positive statements which are made ; but the establishment of the truth of these statements would, of course, lend some support to the author's theory of the decomposition of tissue protein to supply some protective radicle. There is nothing to indicate, however, that any such experiment was ever performed. One can state from experience that to persuade bovines to consume any considerable amount of Matricaria nigellæfolia, while actually giving generous supplementary rations, is a task of very real dfficulty, and any ingenious means by the aid of which it has been accomplished deserve to be recorded.

The writer can speak feelingly on the subject; he can also assert with confidence that the giving of generous rations of the most nutritive foodstuffs available, after the period of experimental feeding, did not appear to affect in any way the development of the intoxication in his own experimental animals, although many of them improved very considerably in condition. In I9I8 a heifer was sent from a farm to the Allerton Laboratory when in a very early stage of a naturally-acquired attack of the intoxication; the animal was in very poor condition, as a result of having had to exist on grazing deficient in both quantity and quality, and it suffered only intermittently, then showing symptoms in a mild form. The heifer was stabled and given the best rations available, including maize meal, bran, lucerne, and sugar-cane. It improved very considerably in condition, but the intoxication continued to develop, until acute symptoms appeared and led fairly rapidly to death.

When we turn to the author's account of the isolation of the active principle and the study of its effects, we note again that no single experimental case is described, even briefly, and we encounter a series of really remarkable statements.

It appears that he was able to exclude the possibility of the active principle being an alkaloid, on account of the acid reaction of the juice of matricaria. One is naturally led to enquire whether all plants known to exert a poisonous action on account of the presence of an alkaloid possess a juice of alkaline reaction, and also whether in the author's opinion the form of combination likely to result from the contact of an alkaloidal base with an acid plant juice is such as must be expected to deprive the alkaloid of its poisonous properties.

The statement in the following paragraph that intoxications caused by free alkaloids, or alkaloids in easily changed form, have a very short incubation period, appears to indicate that the author is not aware of the work that has been done on the alkaloids of certain senecios, or that he has good reason to doubt the connection of those alkaloids with the senecio intoxication.

No definite proof of the existence in the plant of a glucoside is given, and the remarks on the constitution of glucosides are dis- 
tinctly remarkable. The given list of substances which may be united with sugar suggests that alcohols, esters, vegetable acids, etc., are to be distinguished from aromatic ring compounds, and it is surely surprising to find enzymes figuring in a list of substances which may go to make up the glucoside molecule!

The inability of the author to separate the toxin by the use of such agents as sulphuric acid hardly appears to prove the firmness of its combination with some other compound; it seems very probable that the toxin was destroyed and lost during some of these manipulations.

The list of tests for the presence of phenol, after the splitting of the hypothetical glucoside, is hardly convincing when one remembers that the tests were applied to a complex plant extract which had been allowed to ferment. For instance, No. 5 (Millon's reaction) is given not only by phenols, but by a considerable number of other compounds (including proteins and proteoses) which happen to possess a particular grouping.

The presence of phenol in the extracts could certainly have been more satisfactorily demonstrated had the author first subjected them (after acidification) to steam distillation. Even then the presence of phenol could not be assumed necessarily to have the significance which has been assigned to it, and one must regard as very sweeping the statement that carbolic acid produces exactly the same symptoms as are present in pushing disease.

The succeeding statement is even more unexpected: the author boldly asserts that the bovine organism is unable to excrete or otherwise deal with phenol, unlike the other domestic animals and man. No authority is given for this statement, and the writer of this review must confess that he has been unable to trace any work purporting to prove it. If Dr Scharrer himself has performed any experiments which justify this assertion, it is a very great pity that he has not published a detailed account of work of such interest.

Having regard to the fact that animals in general deal with the greater part of the phenol absorbed from the intestine by conjugating it to form phenyl sulphate, one would naturally suppose that the correctness of Dr Scharrer's statement indicated an inability on the part of the bovine organism to bring about this usual reaction. In that case one would have to express surprise that the absence of phenyl sulphate from bovine urine had escaped general notice, for the text-books remark on the relatively high content of ethereal sulphates in herbivorous urine, and merely indicate that the amount is particularly large in equine urine.

Even if we assume that this peculiar behaviour on the part of the bovine organism is a fact which has long escaped notice, but which has now been established, we are confronted subsequently with the statement that the intravenous injection of sodium bisulphite leads to the formation of sodium phenyl sulphate, and that this is excreted in the urine without delay. From this it would appear that the bovine organism is able to carry out perfectly well the usual process of conjugation of phenol, and that the product is readily excreted. If this is the case the nature of the difference between bovines and other mammals, in their behaviour towards phenol, is decidedly obscure. 
If the injection of sodium bisulphite has the effect which is attributed to it, it appears somewhat surprising that it should be necessary to inject it at all in many cases of matricaria intoxication, for in a large number of these cases the liberation of phenol from the glucoside must occur very slowly (if it happens at all), and one knows of no reason to postulate a deficient supply of those sulphur compounds which occur in the animal body as a result of protein decomposition, and which are utilised in the normal production of conjugated sulphates.

Item I7 of the author's summary can only be described as an amazing assertion. If the author feels justified in stating that, "there is no elevation of temperature in pushing disease because phenol is an antipyretic," then one has to suppose that he assumes the presence of some antipyretic agent in every pathological condition not characterised by a rise of body temperature, or at any rate in every such condition which affects the nervous system.

Why should there necessarily be any tendency at all for the temperature to rise? One must confess that one sees no reason to assume that an intoxication of this kind must involve the mechanism regulating the body temperature. Moreover, it may be pointed out that phenol poisoning in both horses and cattle may be accompanied by the development of fever; this fact is demonstrated in the records of cases described by Loft and by Slipper, and quoted by Lander in his Veterinary Toxicology.

Finally, one cannot refrain from remarking that the generic name "matricaria" was certainly not conferred on account of any property or quality possessed by Matricaria nigellæfolia, and it is very unsafe to assume that the pharmacological properties of any particular plant are necessarily. shared by all or any of the other plants belonging to the same genus.

The active principle responsible for the characteristic effects of Matricaria nigellæfolia on the bovine organism may indeed be a glucoside, or a product of the decomposition of a glucoside; but, if that be so, one cannot agree that Dr Scharrer has established the fact. The chemical side of his argument is strewn with obvious errors, and his case as a whole rests on a series of statements which are not supported by any detailed accounts of experiments, and which in some cases are, in the writer's opinion, in direct conflict with known facts.

The paper as a whole strongly suggests the formation of a theory, based on very unsafe foundations, and a subsequent attempt to force the facts to fit it. 